\title{
DEMENZDIAGNOSTIK
}

\section{Reversible Ursachen nicht übersehen!}

Nicht jede Demenz hat eine degenerative oder vaskuläre Ursache, die kausal nicht zu behandeln ist. Bei 5\% aller Demenzerkrankungen liegt eine möglicherweise reversible Ursache zugrunde, wie etwa ein subdurales Hämatom, ein Tumor oder ein Normaldruckhydrocephalus. Um solche potenziell kausal zu behandelnden Erkrankungen nicht zu übersehen, soll bei jeder Demenz, auch wenn etwa klinisch alles auf einen Morbus Alzheimer hin- weist, im Zuge der Differenzialdiagnostik ein konventionelles CT oder ein MRT durchgeführt werden, betonte Prof. Hampel auf dem Jahreskongress der Deutschen Gesellschaft für Psychiatrie, Psychotherapie und Nervenheilkunde in Berlin. Diese Empfehlung ist in der neuen S3Leitlinie Demenzen mit dem Empfehlungsgrad A versehen, d.h. ihr liegt der höchste Evidenzgrad zugrunde. DGPPN-Kongress, Berlin, 24.-27.11.2010

\section{NEUE LIPIDTHERAPIE}

\section{HDL-Cholesterin dramatisch erhöht}

Ein neues Medikament verblüfft mit bis dato kaum für möglich gehaltenen Lipideffekten: Der neue CETP-Hemmer Anacetrapib erhöhte in einer placebokontrollierten Studie das HDL-Cholesterin im Schnitt um 138\% (von 41 mg/dl auf 101 $\mathrm{mg} / \mathrm{dl}$ ). Zugleich wurde das LDL-Cholesterin um $40 \%$ (von $81 \mathrm{mg} / \mathrm{dl}$ auf $45 \mathrm{mg} / \mathrm{dl}$ ) verringert. Diese Effekte waren additiv zu denen einer Statintherapie. In der Studie waren 1623 Patienten mit KHK oder mehreren KHK-Risikofaktoren im Schnitt 18
Monate lang behandelt worden. Dabei gab es keine Hinweise auf ein erhöhtes kardiovaskuläres Risiko. Ob das unter Einfluss von Anacetrapib gebildete HDL-Cholesterin wirklich die funktionellen Eigenschaften des "guten“ Cholesterins hat und dementsprechend die Mortalität und Morbidität zu reduzieren vermag, soll jetzt in einer großen Studie geprüft werden.

N Engl J Med online first, November 2010; doi: 10.1056/NEJMoa1009744

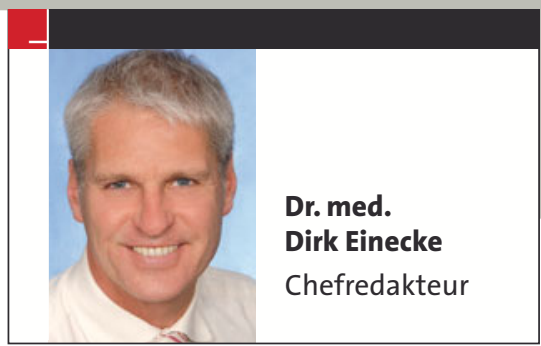

\section{ISCHÄMISCHER SCHLAGANFALL}

\section{Thrombolyse auch für 90-Jährige?}

Ein Alter über 80 Jahren gilt als Kontraindikation für die intravenöse Thrombolyse bei akutem ischämischem Schlaganfall. Zu Unrecht, sagen die Autoren einer Registerstudie mit 29500 Patienten, davon 3472 über 80 Jahren. Zwar war die Prognose der betagten Schlaganfallpatienten erwartungsgemäß schlechter. Aber auch die über 80-Jährigen hatten 90 Tage nach dem Ereignis einen besseren funktionellen Status, wenn sie Alteplase erhalten hatten. Das Alter allein sollte daher kein Ausschlusskriterium für eine medikamentöse Thrombolyse sein, so das Fazit der Studie. Brit Med J 2010;341:c6046

\section{ALTERSMEDIZIN}

\section{Rente hellt die Stimmung auf}

Wer in Rente geht, fällt in ein tiefes Loch, heißt es oft. Eine französische Studie kommt jetzt zu einem ganz anderen Ergebnis. Bei über 11000 Männern und ca. 3000 Frauen wurden über die Zeit von sieben Jahren vor Renteneintritt bis sieben Jahre nach Renteneintritt jährlich bestimmte Krankheiten erfragt. Im Jahr vor der Berentung litten 25\% der Probanden an depressiven Symptomen, $7 \%$ hatten eine chronische Erkrankung. Im ersten Jahr des Ruhestandes besserten sich Erschöpfungszustände signifikant. Auch die Depressionen nahmen ab. Chronische Atemwegserkrankungen, KHK und Diabetes besserten sich hingegen nicht.

Brit Med J 2010, 341:c6149 